\title{
Global attractors for nonlinear wave equations with linear dissipative terms
}

\author{
Zhigang Pan ${ }^{1 *}$, Dongming Yan ${ }^{2 *}$ and Qiang Zhang ${ }^{3}$
}

"Correspondence:

panzhigang@swjtu.edu.cn:

13547895541@126.com

'School of Mathematics, Southwest

Jiaotong University, Chengdu,

610031, China

${ }^{2} \mathrm{School}$ of Mathematics and Statistics, Zhejiang University of Finance and Economics, Hangzhou, 310018, China

Full list of author information is

available at the end of the article

\begin{abstract}
An initial boundary value problem of the semilinear wave equation of which the source term $f(x, u)$ is without variational structure in a bounded domain is considered. Firstly, we prove that it has a unique globally weak solution $\left(u_{1} u_{t}\right) \in C^{0}\left([0, \infty), H_{0}^{1}(\Omega) \times\right.$ $L^{2}(\Omega)$ ) by using our previous results (Pan et al. in Bound. Value Probl. 2012:42, 2012). Secondly, we obtain the existence of global attractors in $H_{0}^{1}(\Omega) \times L^{2}(\Omega)$ by using the $\omega$-limit compactness condition (Ma et al. in Indiana Univ. Math. J. 5(6):1542-1558, 2002), rather than the traditional method.
\end{abstract}

MSC: 35B33; 35B41; 35L71

Keywords: dissipative terms; global attractor; $\omega$-limit compactness

\section{Introduction}

In this paper we are concerned with the existence of global attractors for nonlinear wave equations with linear dissipative terms in a bounded domain $\Omega$ in $R^{n}$ :

$$
\left\{\begin{array}{l}
u_{t t}+2 k u_{t}=\Delta u-|u|^{p-1} u+f(x, u) \quad \text { in } \Omega \times(0, \infty), \\
u(x, t)=0 \quad \text { on } \partial \Omega \times(0, \infty), \\
u(x, 0)=\varphi(x), \quad u_{t}(x, 0)=\psi(x) \quad \text { in } \Omega
\end{array}\right.
$$

where $u_{t}=\frac{\partial u}{\partial t}, u_{t t}=\frac{\partial^{2} u}{\partial t^{2}}, \Delta=\sum_{i=1}^{n} \frac{\partial^{2}}{\partial x_{i}^{2}}, x=\left(x_{1}, \ldots, x_{n}\right)$; the sourcing terms are $-|u|^{p-1} u+$ $f(x, u), 1<p<\frac{n}{n-2}, n \geq 3 ; 1<p<\infty, n=1,2$; and $f(x, u)$ satisfies

$$
|f(x, z)| \leq C|z|^{q}+g(x), \quad q \leq \frac{p+1}{2}, g \in L^{2}(\Omega)
$$

The attractor is an important concept describing the asymptotic properties of dynamical systems. A great deal of work has been devoted to the existence of global attractors of dynamical systems (see, e.g. [1-9] and references therein). The existence of a global attractor (1.1) with a source term only containing $f$ was proved by Hale [7] for $f$ satisfying for $n \geq 3$ the growth condition $f(u) \leq C_{0}\left(|u|^{\gamma}+1\right)$, with $1 \leq \gamma<\frac{n}{n-2}$. For the case $n=2$, Hale and Raugel [10] proved the existence of the attractor under an exponential growth condition of the type $|f(u)| \leq \exp \theta(u)$ (such a condition previously appeared in the work of Gallouët [11]). The existence of the attractor in the critical case $\gamma=\frac{n}{n-2}$ was first proved by Babin and Vishik [1], and then more generally by Arrieta et al. [12]. For other treatments see Chepyzhov and Vishik [3], Ladyzhenskaya [13], Raugel [14] and Temam [9]. When $\Omega$

\section{Springer}

(c) 2015 Pan et al.; licensee Springer. This is an Open Access article distributed under the terms of the Creative Commons Attribution License (http://creativecommons.org/licenses/by/4.0), which permits unrestricted use, distribution, and reproduction in any medium, provided the original work is properly credited. 
is bounded and $u$ is subjected to suitable boundary conditions, the general result is that the dynamical system associated with the problem possesses a global attractor in the natural energy space $H_{0}^{1}(\Omega) \times L^{2}(\Omega)$ if nonlinear term $f$ has a subcritical or critical exponent, because there exist typical parabolic-like flows with an inherent smoothing mechanism. By the traditional method (see [15] for examples), in order to obtain the existence of global attractors for semilinear wave equations, one needs to verify the uniform compactness of the semigroup by getting the boundedness in a more regular function space. However, in some cases it is difficult to obtain the uniform compactness of the semigroup. Fortunately, a new method for obtaining the global attractors has been developed in [16]. With this method, one only needs to verify a necessary compactness condition ( $\omega$-limit compactness) with the same type of energy estimates as those for establishing the absorbing sets. In this paper, we use this method to obtain the existence of global attractors for problem (1.1) with the general condition where the source term $f(x, u)$ is without variational structure.

This paper is organized as follows:

- in Section 2 we recall some preliminary tools, definitions and our previous results;

- in Section 3 we obtain the existence and uniqueness of weak solution by using our previous results [17] and the various conditions can also be found [18];

- in Section 4 we obtain our main results for problem (1.1) by using the new method ( $\omega$-compactness condition).

\section{Preliminaries}

Consider the abstract nonlinear evolution equation defined on $X$, given by

$$
\left\{\begin{array}{l}
\frac{d^{2} u}{d t^{2}}+k \frac{d u}{d t}=G(u), \quad k>0 \\
u(x, 0)=\varphi(x) \\
u_{t}(x, 0)=\psi(x)
\end{array}\right.
$$

where $G: X_{2} \times \mathbf{R}^{+} \rightarrow X_{1}{ }^{*}$ is a mapping, $X_{2} \subset X_{1}, X_{1}, X_{2}$ are Banach spaces and $X_{1}^{*}$ is the dual space of $X_{1}, \mathbf{R}^{+}=[0, \infty), u=u(x, t)$ is an unknown function.

First we introduce a sequence of function spaces:

$$
\left\{\begin{array}{l}
X \subset H_{2} \subset X_{2} \subset X_{1} \subset H, \\
X_{2} \subset H_{1} \subset H,
\end{array}\right.
$$

where $H, H_{1}, H_{2}$ are Hilbert spaces, $X$ is a linear space, $X_{1}, X_{2}$ are Banach spaces and all inclusions are dense embeddings.

Suppose that

$$
\left\{\begin{array}{l}
L: X \rightarrow X_{1} \text { is a one to one dense linear operator, } \\
\langle L u, v\rangle_{H}=\langle u, v\rangle_{H_{1}}, \quad \forall u, v \in X
\end{array}\right.
$$

In addition, the operator $L$ has an eigenvalue sequence

$$
L e_{k}=\lambda_{k} e_{k} \quad(k=1,2, \ldots)
$$

such that $\left\{e_{k}\right\} \subset X$ is the common orthogonal basis of $H$ and $H_{2}$. 
Definition 2.1 [17] Set $(\varphi, \psi) \in X_{2} \times H_{1}, u \in W_{\text {loc }}^{1, \infty}\left((0, \infty), H_{1}\right) \cap L_{\text {loc }}^{\infty}\left((0, \infty), X_{2}\right)$ is called a globally weak solution of (2.1), if $\forall v \in X_{1}$, we have

$$
\left\langle u_{t}, v\right\rangle_{H}+k\langle u, v\rangle_{H}=\int_{0}^{t}\langle G u, v\rangle d t+k\langle\varphi, v\rangle_{H}+\langle\psi, v\rangle_{H} .
$$

Definition 2.2 [17] Let $Y_{1}, Y_{2}$ be Banach spaces, the solution $u(t, \varphi, \psi)$ of (2.1) is called uniformly bounded in $Y_{1} \times Y_{2}$, if for any bounded domain $\Omega_{1} \times \Omega_{2} \subset Y_{1} \times Y_{2}$, there exists a constant $C$ which only depends on the domain $\Omega_{1} \times \Omega_{2}$, such that

$$
\|u\|_{Y_{1}}+\left\|u_{t}\right\|_{Y_{2}} \leq C, \quad \forall(\varphi, \psi) \in \Omega_{1} \times \Omega_{2} \text { and } t \geq 0
$$

Suppose that $G=A+B: X_{2} \times \mathbf{R}^{+} \rightarrow X_{1}{ }^{*}$. Throughout this paper, we assume that:

(i) There exists a functional $F \in C^{1}: X_{2} \rightarrow \mathbf{R}^{1}$ such that

$$
\langle A u, L v\rangle=\langle-D F(u), v\rangle, \quad \forall u, v \in X
$$

(ii) The functional $F$ is coercive, i.e.

$$
F(u) \rightarrow \infty \quad \Leftrightarrow \quad\|u\|_{X_{2}} \rightarrow \infty .
$$

(iii) There exist constants $C_{1}>0$ and $C_{2}>0$ such that

$$
|\langle B u, L v\rangle| \leq C_{1} F(u)+C_{2}\|v\|_{H_{1}}^{2}, \quad \forall u, v \in X
$$

Lemma 2.1 [17] Set $G: X_{2} \times \mathbf{R}^{+} \rightarrow X_{1}{ }^{*}$ to be weakly continuous, $(\varphi, \psi) \in X_{2} \times H_{1}$, then we obtain the following results:

(1) If $G=A$ satisfies the assumptions (i) and (ii), then there exists a globally weak solution of (2.1),

$$
u \in W_{\mathrm{loc}}^{1, \infty}\left((0, \infty), H_{1}\right) \cap L_{\mathrm{loc}}^{\infty}\left((0, \infty), X_{2}\right)
$$

and $u$ is uniformly bounded in $X_{2} \times H_{1}$.

(2) If $G=A+B$ satisfies the assumptions (i), (ii) and (iii), then there exists a globally weak solution of $(2.1)$,

$$
u \in W_{\mathrm{loc}}^{1, \infty}\left((0, \infty), H_{1}\right) \cap L_{\mathrm{loc}}^{\infty}\left((0, \infty), X_{2}\right) .
$$

(3) Furthermore, if $G=A+B$ satisfies

$$
|\langle G u, v\rangle| \leq \frac{1}{2}\|v\|_{H}^{2}+C F(u)+g(t)
$$

for some $g \in L_{\mathrm{loc}}^{1}(0, \infty)$, then $u \in W_{\mathrm{loc}}^{2,2}((0, \infty), H)$.

A family of operators $S(t): X \rightarrow X(t \geq 0)$ is called a semigroup generated by (2.1) if it satisfies the following properties: 
(1) $S(t): X \rightarrow X$ is a continuous map for any $t \geq 0$,

(2) $S(0)=\mathrm{id}: X \rightarrow X$ is the identity,

(3) $S(t+s)=S(t) \cdot S(s), \forall t, s \geq 0$. Then the solution of (2.1) can be expressed as

$$
u\left(t, u_{0}\right)=S(t) u_{0}
$$

Introducing the expression of the abstract semilinear wave equation:

$$
\left\{\begin{array}{l}
\frac{d^{2} u}{d t^{2}}+2 k \frac{d u}{d t}=L u+T(u), \quad k \geq 0 \\
u(x, 0)=\varphi(x) \\
u_{t}(x, 0)=\psi(x)
\end{array}\right.
$$

where $X_{1}, X$ are Banach spaces, $X_{1} \subset X$ is a dense inclusion, $L: X_{1} \rightarrow X$ is a sectorial linear operator, and $T: X_{1} \rightarrow X$ is a nonlinear bounded operator.

Lemma 2.2 [19] Set $L: X_{1} \rightarrow X$, a sectorial linear operator and $T: X_{1} \rightarrow X$, a nonlinear bounded operator, $\mathcal{L}=L+k^{2} I$, then the solution of (2.9) can be expressed as follows:

$$
\begin{aligned}
u= & e^{-k t}\left[\cos t(-\mathcal{L})^{\frac{1}{2}} \varphi+k(-\mathcal{L})^{-\frac{1}{2}} \sin (-\mathcal{L})^{\frac{1}{2}} \varphi+(-\mathcal{L})^{-\frac{1}{2}} \sin t(-\mathcal{L})^{\frac{1}{2}} \psi\right. \\
& \left.+\int_{0}^{t} e^{-k(t-\tau)}(-\mathcal{L})^{-\frac{1}{2}} \sin (t-\tau)(-\mathcal{L})^{\frac{1}{2}} T(u) d \tau\right] \\
u_{t}= & -k u+e^{-k t}\left[-(-\mathcal{L})^{\frac{1}{2}} \sin t(-\mathcal{L})^{\frac{1}{2}} \varphi+k \cos t(-\mathcal{L})^{\frac{1}{2}} \varphi+\cos t(-\mathcal{L})^{\frac{1}{2}} \psi\right. \\
& \left.+\int_{0}^{t} e^{-k(t-\tau)} \cos (t-\tau)(-\mathcal{L})^{\frac{1}{2}} T(u) d \tau\right]
\end{aligned}
$$

Next, we introduce the concepts and definitions of invariant sets, global attractors, and $\omega$-limit compactness sets for the semigroup $S(t)$.

Definition 2.3 Let $S(t)$ be a semigroup defined on $X$. A set $\Sigma \subset X$ is called an invariant set of $S(t)$ if $S(t) \Sigma=\Sigma, \forall t \geq 0$. An invariant set $\Sigma$ is an attractor of $S(t)$ if $\Sigma$ is compact, and there exists a neighborhood $U \subset X$ of $\Sigma$ such that, for any $u_{0} \in U$,

$$
\inf _{v \in \Sigma}\left\|S(t) u_{0}-v\right\|_{X} \rightarrow 0, \quad \text { as } t \rightarrow 0
$$

In this case, we say that $\Sigma$ attracts $U$. Especially, if $\Sigma$ attracts any bounded set of $X, \Sigma$ is called a global attractor of $S(t)$ in $X$.

Definition 2.4 Let $X$ be an infinite dimensional Banach space and $A$ be a bounded subset of $X$. The measure of noncompactness $\gamma(A)$ of $A$ is defined by

$$
\gamma(A)=\inf \{\delta>0 \mid \text { for } A \text { there exists a finite cover by sets whose diameter } \leq \delta\}
$$

Lemma 2.3 [11] If $A_{n} \subset X$ is a sequence bounded and closed sets, $A_{n} \neq \emptyset, A_{n+1} \subset A_{n}$, and $\gamma\left(A_{n}\right) \rightarrow 0(n \rightarrow \infty)$, then the set $A=\bigcap_{n=1}^{\infty} A_{n}$ is a nonempty compact set. 
Definition 2.5 [16] A semigroup $S(t): X \rightarrow X(t \geq 0)$ in $X$ is called $\omega$-limit compact, if for any bounded set $B \subset X$ and $\forall \varepsilon>0$, there exists $t_{0}$ such that

$$
\gamma\left(\bigcup_{t \geq t_{0}} S(t) B\right) \leq \varepsilon
$$

where $\gamma$ is a noncompact measure in $X$.

For a set $D \subset X$, we define the $\omega$-limit set of $D$ as follows:

$$
\omega(D)=\bigcap_{s \geq 0} \overline{\bigcup_{t \geq s} S(t) D}
$$

where the closure is taken in the $X$-norm.

Lemma 2.4 [19] Let $S(t)$ be a semigroup in $X$, then $S(t)$ has a global attractor $\mathcal{A}$ in $X$ if and only if

(1) $S(t)$ has $\omega$-limit compactness, and

(2) there is a bounded absorbing set $B \subset X$.

In addition, the $\omega$-limit set of $B$ is the attractor $\mathcal{A}=\omega(B)$.

Remark 2.1 Although the lemma has been proved partly in [19], we still give a proof here. Our proof is different from that in [20] but is similar to that in [16]. We adopt and present the proof also because we will use the same method to obtain the existence of the global attractor.

Proof Step 1. To prove the sufficiency of Lemma 2.4.

(a) $S(t)$ has $\omega$-limit compactness, i.e., for any bounded set $B \subset X$ and $\forall \varepsilon>0$, there exists a $t_{0}$, such that

$$
\gamma\left(\bigcup_{t \geq t_{0}} S(t) B\right) \leq \varepsilon
$$

So, we know that $\omega(B)=\bigcap_{t_{0}=0}^{\infty} \overline{\bigcup_{t \geq t_{0}} S(t) B}$ is a compact set from Lemma 2.3.

(b) $\omega(B)$ is nonempty.

For $B \neq \emptyset$, so $\overline{\bigcup_{t \geq s} S(t) B} \neq \emptyset, \forall s \geq 0$, and

$$
\overline{\bigcup_{t \geq s_{1}} S(t) B} \subset \overline{\bigcup_{t \geq s_{2}} S(t) B}, \quad \forall s_{1} \geq s_{2}
$$

we can obtain

$$
\omega(B)=\bigcap_{s \geq 0}^{\infty} \overline{\bigcup_{t \geq s} S(t) B} \neq \emptyset .
$$

(c) $\omega(B)$ is invariant.

For $x \in \omega(B) \Leftrightarrow$ there exist $\left\{x_{n}\right\} \in B$ and $t_{n} \rightarrow \infty$, such that $S\left(t_{n}\right) x_{n} \rightarrow x$.

If $y \in S(t) \omega(B)$, then for some $x \in \omega(B), y=S(t) x$. 
Hence, there exist $\left\{x_{n}\right\} \subset B, t_{n} \rightarrow \infty$, such that

$$
S(t) S\left(t_{n}\right) x_{n}=S\left(t+t_{n}\right) x_{n} \rightarrow S(t) x=y .
$$

In conclusion, $y \in \omega(B), S(t) \omega(B) \in \omega(B), \forall t \geq 0$.

If $x \in \omega(B)$, fix $\left\{x_{n}\right\} \subset B$ and $t_{n}$, such that

$$
S(t) x_{n} \rightarrow x, \quad \text { as } t_{n} \rightarrow \infty, n \rightarrow \infty .
$$

$S(t)$ is $\omega$-limit compact, i.e., there exists a $y \in H$, such that

$$
S(t) \bigcap_{t_{n} \geq 0} \overline{\bigcup_{t \geq t_{n}} S\left(t_{n}\right) x_{n}} \rightarrow y, \quad n \rightarrow \infty .
$$

Therefore $y \in \omega(B)$.

For

$$
\bigcap_{t_{n} \geq 0} \overline{\bigcup_{t \geq t_{n}} S\left(t_{n}\right) x_{n}}=\bigcap_{t_{n} \geq 0} \overline{\bigcup_{t \geq t_{n}} S(t) S\left(t_{n}-t\right) x_{n}} \rightarrow \bigcap_{t_{n} \geq 0} \overline{\bigcup_{t \geq t_{n}} S(t) y}
$$

and

$$
S\left(t_{n}\right) x_{n} \rightarrow x \in \omega(B)
$$

which implies that

$$
S(t) y \rightarrow x, \quad \omega(B) \subset S(t) \omega(B) .
$$

In conclusion, combining (a)-(c) and condition (2), Step 1 has been proved.

Step 2 . To prove the necessity of Lemma 2.4 .

If $\mathcal{A}$ is a global attractor, then the $\varepsilon$-neighborhood $U_{\varepsilon}(\mathcal{A}) \subset X$ is an absorbing set. So we need only to prove $S(t)$ has $\omega$-limit compactness.

Since $U_{\varepsilon}(\mathcal{A})$ is an absorbing set, for any bounded set $B \subset X$ and $\varepsilon>0$, there exists a time $t_{\varepsilon}(B)>0$ such that

$$
\bigcup_{t \geq t_{\varepsilon}(B)} S(t) B \subset U_{\frac{\varepsilon}{4}}(\mathcal{A})=\left\{x \in X \mid \operatorname{dist}(x, \mathcal{A})<\frac{\varepsilon}{4}\right\} .
$$

On the other hand, $\mathcal{A}$ is a compact set, and there exist finite elements $x_{1}, x_{2}, \ldots, x_{n} \in X$ such that

$$
\mathcal{A} \subset \bigcup_{k=1}^{n} U\left(x_{k}, \frac{\varepsilon}{4}\right)
$$

Then

$$
U_{\frac{\varepsilon}{2}}(\mathcal{A}) \subset \bigcup_{k=1}^{n} U\left(x_{k}, \frac{\varepsilon}{2}\right),
$$


which implies that

$$
\gamma\left(\bigcup_{t \geq t_{\varepsilon}(B)} S(t) B\right) \leq \gamma\left(U_{\frac{\varepsilon}{4}}(\mathcal{A})\right) \leq \varepsilon .
$$

Hence, Lemma 2.4 has been proved.

\section{Existence and uniqueness of globally weak solution}

Now, in this section, we begin to prove that problem (1.1) has a unique globally weak solution $\left(u, u_{t}\right) \in C^{0}\left([0, \infty), H_{0}^{1} \times L^{2}(\Omega)\right)$.

Theorem 3.1 (Existence) If $\forall(\varphi, \psi) \in H_{0}^{1}(\Omega) \times L^{2}(\Omega)$, f satisfies condition (1.2) and $1<p<$ $\frac{n}{n-2}, n \geq 3 ; 1<p<\infty, n=1,2$, then (1.1) has a globally weak solution

$$
u \in W_{\mathrm{loc}}^{1, \infty}\left((0, \infty), L^{2}(\Omega)\right) \cap L_{\mathrm{loc}}^{\infty}\left((0, \infty), H_{0}^{1}(\Omega)\right) .
$$

Remark 3.1 Divide the operator $G(u)$ in Lemma 2.1 into two parts: $A$ and $B$, where $A$ has a variational structure and $B$ has a non-variational structure. Then we obtain the globally weak solution by applying our result (2) in Lemma 2.1.

Proof Fix spaces as follows:

$$
\begin{aligned}
& X_{2}=X_{1}=H_{0}^{1}(\Omega) \cap L^{p+1}(\Omega), \\
& X=C_{0}^{\infty}(\Omega), \quad H_{1}=H=L^{2}(\Omega) .
\end{aligned}
$$

In problem (1.1), set $G(u)=\Delta u-|u|^{p-1} u+f(x, u)$.

Define the map $G(u)=A+B: X_{1} \rightarrow X_{1}^{*}$ as

$$
\begin{aligned}
& \langle A u, v\rangle=-\int_{\Omega}\left[\nabla u \cdot \nabla v+|u|^{p-1} u \cdot v\right] d x \\
& \langle B u, v\rangle=\int_{\Omega} f(x, u) v d x
\end{aligned}
$$

Note the functional $I: X_{1} \rightarrow R^{1}$,

$$
I[u]=\int_{\Omega}\left[\frac{1}{2}|\nabla u|^{2}+\frac{1}{p+1}|u|^{p+1}\right] d x .
$$

Obviously, we obtain

$$
\langle A u, v\rangle=-\langle D I[u], v\rangle, \quad \forall u, v \in X
$$

and

$$
I[u] \rightarrow \infty \quad \Leftrightarrow \quad\|u\|_{X_{1}} \rightarrow \infty,
$$

which implies that conditions (1) and (2) in Lemma 2.1 hold. 
From the growth restriction condition (1.2), we get

$$
\begin{aligned}
|\langle B u, v\rangle| & =\left|\int_{\Omega} f(x, u) v d x\right| \\
& \leq \int_{\Omega}|f(x, u)||v| d x \\
& \leq \frac{1}{2} \int_{\Omega}|v|^{2} d x+\frac{1}{2} \int_{\Omega}|f(x, u)|^{2} d x \\
& \leq \frac{1}{2} \int_{\Omega}|v|^{2} d x+C \int_{\Omega}\left[|u|^{2 q}+g^{2}(x)\right] d x \\
& \leq \frac{1}{2}\|v\|_{H_{1}}^{2}+C_{1} \int_{\Omega}|u|^{p+1} d x+C_{2} \\
& \leq \frac{1}{2}\|v\|_{H_{1}}^{2}+C_{1} I[u]+C_{2},
\end{aligned}
$$

where $C, C_{1}, C_{2}>0$. It implies that condition (3) in Lemma 2.1 holds.

In conclusion, we see that problem (1.1) has a globally weak solution

$$
u \in W_{\mathrm{loc}}^{1, \infty}\left((0, \infty), L^{2}(\Omega)\right) \cap L_{\mathrm{loc}}^{\infty}\left((0, \infty), H_{0}^{1}(\Omega)\right)
$$

from the second result in Lemma 2.1.

Next, we prove the uniqueness of the globally weak solution to problem (1.1).

Theorem 3.2 If $u \in W_{\text {loc }}^{1, \infty}\left((0, \infty), L^{2}(\Omega)\right) \cap L_{\text {loc }}^{\infty}\left((0, \infty), H_{0}^{1}(\Omega)\right)$ is a weak solution of problem (1.1), then the solution $u$ is unique.

Remark 3.2 From the formula of the wave equation in Lemma 2.2 and using the Gronwall inequality, we obtain the uniqueness of the globally weak solution.

Proof Set $u_{1}, u_{2} \in W_{\mathrm{loc}}^{1, \infty}\left((0, \infty), L^{2}(\Omega)\right) \cap L_{\mathrm{loc}}^{\infty}\left((0, \infty), H_{0}^{1}(\Omega)\right)$ as the solutions of problem (1.1), then from Lemma 2.2, we get $u_{i} \in C^{0}\left([0, \infty), H_{0}^{1}(\Omega)\right), i=1,2$, and

$$
\begin{aligned}
\left\|u_{1}-u_{2}\right\|_{H_{0}^{1}} & =\left\|\left(-\triangle^{\frac{1}{2}}\right)\left(u_{1}-u_{2}\right)\right\|_{L^{2}} \\
& \leq C \int_{0}^{t}\left\|\left[\left|u_{2}\right|^{p-1} u_{2}-\left|u_{1}\right|^{p-1} u_{1}\right]+\left[f\left(x, u_{1}\right)-f\left(x, u_{2}\right)\right]\right\|_{L^{2}} d \tau \\
& \leq C_{1} \int_{0}^{t}\left[\left(\left\||\tilde{u}|^{p-1}\right\|+\|D f(x, \tilde{u})\|\right) \cdot\left\|u_{1}-u_{2}\right\|_{H_{0}^{1}}\right] d \tau ;
\end{aligned}
$$

by using the Gronwall inequality, we easily obtain

$$
\left\|u_{1}-u_{2}\right\|_{H_{0}^{1}} \leq 0
$$

where $\tilde{u}$ is the mean value between $u_{1}$ and $u_{2}$.

It implies that

$$
\left\|u_{1}-u_{2}\right\|_{H_{0}^{1}} \leq 0 \quad \Rightarrow \quad u_{1}=u_{2}
$$




\section{Existence of global attractor}

In this section, we proved the existence of global attractor to problem (1.1).

Theorem 4.1 For any $(\varphi, \psi) \in\left(H_{0}^{1}(\Omega) \times L^{2}(\Omega)\right)$, the sourcing term $f$ satisfies the growth restriction (1.2) and the exponent of $p$ satisfies $1<p<\frac{n}{n-2}, n \geq 3$ or $1<p<\infty, n=1,2$; then problem (1.1) has a global attractor $\mathcal{A}$ in $\left(H_{0}^{1}(\Omega) \times L^{2}(\Omega)\right)$.

Remark 4.1 Comparing Remark 3.1, we divide the operator $G(u)$ of (2.1) into two parts: $L$ and $T$, where $L$ is a linear operator, while $T$ is a nonlinear operator. We obtain the global attractor of problem (1.1) by using Lemma 2.4 .

Proof According to Lemma 2.4, we prove Theorem 4.1 in the following three steps.

Step 1. Problem (1.1) has a globally unique weak solution.

Step 2. $S(t)$ has a bounded absorbing set in $H_{0}^{1}(\Omega) \times L^{2}(\Omega)$.

From Theorems 3.1 and 3.2, we see that problem (1.1) has a globally unique weak solution $\left(u, u_{t}\right) \in C^{0}\left([0, \infty), H_{0}^{1} \times L^{2}\right)$. Equation (1.1) generates a semigroup:

$$
S(t): H_{\frac{1}{2}} \times H \rightarrow H_{\frac{1}{2}} \times H .
$$

Fix the spaces as follows:

$$
\begin{aligned}
& H=L^{2}(\Omega), \quad H_{1}=H^{2}(\Omega) \cap H_{0}^{1}(\Omega), \\
& L: H_{1} \rightarrow H, \quad T: H_{1} \rightarrow H .
\end{aligned}
$$

Note that

$$
\begin{aligned}
& L u=\Delta u, \\
& T u=-|u|^{p-1} u+f(x, u),
\end{aligned}
$$

and $L$ generates the fractional space, $H_{\frac{1}{2}}=H_{0}^{1}(\Omega)$.

Obviously, there exists a $C^{1}$ functional $F: H_{\frac{1}{2}} \rightarrow R^{1}$ such that

$$
F(u)=\frac{1}{p+1}|u|^{p+1}-\int_{0}^{t} f(x, u) d \tau,
$$

and we easily get

$$
T(u)=-D F(u), \quad \forall u \in H_{1} .
$$

Since

$$
|f(x, z)| \leq C|z|^{q}+g(x), \quad q \leq \frac{p+1}{2}, g \in L^{2}(\Omega)
$$

then we get

$$
F(u) \geq-C_{1}
$$


and

$$
\langle D F(u), u\rangle_{H}-k\langle u, v\rangle_{H} \geq-\frac{1}{2}\|v\|_{H}^{2}-C_{2}, \quad C_{2}>0 .
$$

Equation (1.1) is equivalent to the equations that follow:

$$
\left\{\begin{array}{l}
\frac{\partial u}{\partial t}=-k u+v, \quad k \geq 0 \\
\frac{\partial v}{\partial t}=L u+k^{2} u-k v-|u|^{p-1} u+f(x, u) .
\end{array}\right.
$$

Multiply $(4.7)$ by $(-L u, v)$ and take the inner product in $H$ :

$$
\begin{aligned}
& \left\langle\frac{\partial u}{\partial t},-L u\right\rangle_{H}=-k\langle u,-L u\rangle_{H}+\langle-L u, v\rangle_{H}, \\
& \left\langle\frac{\partial v}{\partial t}, v\right\rangle_{H}=\langle L u, v\rangle_{H}+\left\langle k^{2} u, v\right\rangle_{H}-k\langle v, v\rangle_{H}+\langle T(u), v\rangle_{H}
\end{aligned}
$$

Summing (4.8) and (4.9), it follows that

$$
\begin{aligned}
& \left\langle\frac{\partial u}{\partial t},-L u\right\rangle_{H}+\left\langle\frac{\partial v}{\partial t}, v\right\rangle_{H} \\
& \quad=-k\langle u,-L u\rangle_{H}-k\langle v, v\rangle_{H}+k^{2}\langle u, v\rangle_{H}+\langle T u, v\rangle_{H} .
\end{aligned}
$$

Furthermore,

$$
\langle-L u, \omega\rangle_{H}=\left\langle\left(-L^{\frac{1}{2}}\right) u,\left(-L^{\frac{1}{2}}\right) \omega\right\rangle_{H}, \quad \forall u, \omega \in H_{\frac{1}{2}} .
$$

From (4.4) and (4.7), we get

$$
\begin{aligned}
\langle T u, v\rangle_{H} & =\left\langle T u, \frac{\partial u}{\partial t}+k u\right\rangle_{H} \\
& =\left\langle-D F(u), \frac{\partial u}{\partial t}+k u\right\rangle_{H} \\
& =-\left\langle D F(u), \frac{\partial u}{\partial t}\right\rangle_{H}-k\langle D F(u), u\rangle_{H} \\
& =-\frac{d F(u)}{d t}-k\langle D F(u), u\rangle_{H}
\end{aligned}
$$

Integrating (4.10) over $[0, t]$ with respect to time $t$ and combining the two formulas, we get

$$
\begin{aligned}
\frac{1}{2} & \|u\|_{H_{\frac{1}{2}}^{2}}+\frac{1}{2}\|v\|_{H}^{2}-\frac{1}{2}\|\varphi\|_{H_{\frac{1}{2}}^{2}}-\frac{1}{2}\|\psi\|_{H^{2}} \\
& =\int_{0}^{t}\left[\left\langle\frac{\partial u}{\partial t},-L u\right\rangle_{H}+\left\langle\frac{\partial v}{\partial t}, v\right\rangle_{H}\right] d \tau \\
& =-k \int_{0}^{t}\left[\langle u,-L u\rangle_{H}+\langle v, v\rangle_{H}-k\langle u, v\rangle_{H}\right] d \tau+\int_{0}^{t}\langle T u, v\rangle_{H} d \tau
\end{aligned}
$$




$$
\begin{aligned}
= & -k \int_{0}^{t}\left[\left\langle\left(-L^{\frac{1}{2}}\right) u,\left(-L^{\frac{1}{2}}\right) u\right\rangle_{H}+\|v\|_{H}^{2}-k\langle u, v\rangle_{H}\right] d \tau \\
& +\int_{0}^{t}\left[-\frac{d F(u)}{d t}-k\langle D F(u), u\rangle_{H}\right] d \tau \\
= & -k \int_{0}^{t}\left[\|u\|_{H_{\frac{1}{2}}^{2}}+\|v\|_{H}^{2}-k\langle u, v\rangle_{H}\right] d \tau-F(u(t))+F(u(0)) \\
& -k \int_{0}^{t}\langle D F(u), u\rangle_{H} d \tau \\
= & -k \int_{0}^{t}\left[\|u\|_{H_{\frac{1}{2}}^{2}}+\langle D F(u), u\rangle_{H}-k\langle u, v\rangle_{H}\right] d \tau-F(u)+F(\varphi) ;
\end{aligned}
$$

combining with (4.6), it follows that

$$
\|u\|_{H_{\frac{1}{2}}^{2}}+\|v\|_{H}^{2} \leq-k \int_{0}^{t}\left[\|u\|_{H_{\frac{1}{2}}^{2}}+\|v\|_{H}^{2}\right] d \tau+f(\varphi, \psi)+C t, \quad C>0 .
$$

Applying the Gronwall inequality, we get

$$
\|u\|_{H_{\frac{1}{2}}^{2}}+\|v\|_{H}^{2} \leq f(\varphi, \psi) e^{-k t}+C_{1}\left(1-e^{-t}\right) .
$$

It implies that $S(t)$ has a bounded absorbing set in $H_{\frac{1}{2}} \times H$.

Step 3. $S(t)$ has $\omega$-limit compactness.

From the formula in Lemma 2.2, the solution of problem (1.1) can be expressed as follows:

$$
\begin{aligned}
u= & e^{-k t}\left[\cos t(-\triangle)^{\frac{1}{2}} \varphi+k(-\triangle)^{-\frac{1}{2}} \sin t(-\triangle)^{\frac{1}{2}} \varphi+(-\triangle)^{-\frac{1}{2}} \sin t(-\triangle)^{\frac{1}{2}} \psi\right] \\
& +\int_{0}^{t}\left[e^{-k(t-\tau)}(-\triangle)^{-\frac{1}{2}} \sin (t-\tau)(-\triangle)^{\frac{1}{2}}\left(-|u|^{p-1} u+f\right)\right] d \tau, \\
u_{t}= & -k u+e^{-k t}\left[-(-\triangle)^{\frac{1}{2}} \sin t(-\triangle)^{\frac{1}{2}} \varphi+k \cos t(-\triangle)^{\frac{1}{2}} \varphi+\cos t(-\triangle)^{\frac{1}{2}} \psi\right] \\
& +\int_{0}^{t}\left[e^{-k(t-\tau)} \cos (t-\tau)(-\triangle)^{\frac{1}{2}}\left(-|u|^{p-1} u+f\right)\right] d \tau .
\end{aligned}
$$

Since the linear operator

$$
L=\triangle: H^{2}(\Omega) \times H_{0}^{1}(\Omega) \rightarrow L^{2}(\Omega)
$$

is a symmetrical sector operator, it has the eigenvalue sequence:

$$
0>\lambda_{1} \geq \lambda_{2} \geq \cdots, \quad \lambda_{k} \rightarrow-\infty, k \rightarrow \infty .
$$

Then

$$
\begin{aligned}
& \sin t(-\triangle)^{\frac{1}{2}} v=\sum_{j=1}^{\infty} v_{j} \sin \sqrt{-\lambda_{j}} t e_{j}, \\
& \cos t(-\triangle)^{\frac{1}{2}} v=\sum_{j=1}^{\infty} v_{j} \cos \sqrt{-\lambda_{j}} t e_{j} .
\end{aligned}
$$


For any $v=\sum_{j=1}^{\infty} v_{j} e_{j} \in L^{2}(\Omega)$ and $-\lambda_{j}>0(j \geq 1)$, the operator

$$
\sin t(-\triangle)^{\frac{1}{2}}, \cos t(-\triangle)^{\frac{1}{2}}: L^{2}(\Omega) \rightarrow L^{2}(\Omega)
$$

is uniformly bounded, i.e.

$$
\left\|\sin t(-\triangle)^{\frac{1}{2}}\right\|_{L^{2}},\left\|\cos t(-\triangle)^{\frac{1}{2}}\right\|_{L^{2}} \leq 1, \quad \forall t \geq 0
$$

Furthermore, $\left(u, u_{t}\right)$ contains two parts:

degenerative term

$$
\left(\begin{array}{l}
u^{1} \\
u_{t}^{1}
\end{array}\right)=e^{-k t}\left(\begin{array}{cc}
\cos (-\triangle)^{\frac{1}{2}}+k(-\triangle)^{-\frac{1}{2}} \sin t(-\triangle)^{\frac{1}{2}} & (-\triangle)^{-\frac{1}{2}} \sin t(-\triangle)^{\frac{1}{2}} \\
k \cos t(-\triangle)^{\frac{1}{2}}-(-\triangle)^{\frac{1}{2}} \sin t(-\triangle)^{\frac{1}{2}} & \cos t(-\triangle)^{\frac{1}{2}}
\end{array}\right)\left(\begin{array}{l}
\varphi \\
\psi
\end{array}\right)
$$

integral term

$$
\left(\begin{array}{c}
u^{2} \\
u_{t}^{2}
\end{array}\right)=\left(\begin{array}{c}
\int_{0}^{t} e^{-k(t-\tau)}(-\triangle)^{-\frac{1}{2}} \sin (t-\tau)(-\triangle)^{\frac{1}{2}}\left(-|u|^{p-1} u+f\right) d \tau \\
\int_{0}^{t} e^{-k(t-\tau)} \cos (t-\tau)(-\triangle)^{\frac{1}{2}}\left(-|u|^{p-1} u+f\right) d \tau
\end{array}\right)
$$

From the uniformly bounded condition (4.17), we get

$$
\lim _{t \rightarrow \infty}\left(u_{1}, u_{t}^{1}\right)=0 \quad \text { in } H_{0}^{1}(\Omega) \times L^{2}(\Omega)
$$

and for any $(\varphi, \psi) \in B$,

$$
\bigcup_{t \geq 0}\left(u^{2}, u_{t}^{2}\right) \text { is a compact set in } H_{0}^{1}(\Omega) \times L^{2}(\Omega)
$$

where $B \subset H_{0}^{1}(\Omega) \times L^{2}(\Omega)$ is a bounded set.

From $(1.2)$ and $H_{0}^{1}(\Omega) \hookrightarrow L^{2 p}(\Omega)\left(p<\frac{n}{n-2}\right)$, we get

$$
T: H_{0}^{1}(\Omega) \rightarrow L^{2}(\Omega) \text { is a compact map, }
$$

Hence, combining (4.18) and (4.19), for the noncompact measure $\gamma$ we get

$$
\begin{aligned}
& \gamma\left(\bigcup_{t \geq t_{0}} S(t) B\right) \\
& \quad=\gamma\left(\bigcup_{t \geq t_{0}}\left(u(t, B), u_{t}(t, B)\right)\right) \\
& \quad \leq \gamma\left(\bigcup_{t \geq t_{0}}\left(u^{1},-k u^{1}+u_{t}^{1}\right)\right)+\gamma\left(\bigcup_{t \geq t_{0}}\left(u^{2},-k u^{2}+u_{t}^{2}\right)\right) \\
& \quad=\gamma\left(\bigcup_{t \geq t_{0}}\left(u^{1},-k u^{1}+u_{t}^{1}\right)\right) \\
& \quad \rightarrow 0 \quad\left(t_{0} \rightarrow \infty\right)
\end{aligned}
$$


it implies that

\section{$S(t)=\left(u(t, \cdot), u_{t}(t, \cdot)\right)$ has $\omega$-limit compactness.}

Finally, combining Step 2 and Step 3, applying Lemma 2.4, problem (1.1) has a global attractor $\mathcal{A}$ in $H_{0}^{1}(\Omega) \times L^{2}(\Omega)$.

\section{Competing interests}

The authors declare that they have no competing interests.

\section{Authors' contributions}

DY and QZ discussed with ZP the paper who helped to check and prove the whole paper. All authors read and approved the final manuscript.

\section{Author details}

'School of Mathematics, Southwest Jiaotong University, Chengdu, 610031, China. ${ }^{2}$ School of Mathematics and Statistics, Zhejiang University of Finance and Economics, Hangzhou, 310018, China. ${ }^{3}$ School of Computer Science, Civil Aviation Flight University of China, Guanghan, 618307, China.

\section{Acknowledgements}

The authors are grateful to professor Tian Ma for his helpful comments. This work is supported by the Fundamental Research Funds for the Central Universities (No. 2682014BR036)

Received: 26 September 2014 Accepted: 26 December 2014 Published online: 30 January 2015

\section{References}

1. Babin, AV, Vishik, Ml: Attractors of Evolution Equations. Nauka, Moscow (1989); English translation: North-Holland, Amsterdam (1992)

2. Ball, JM: Global attractors for damped semilinear wave equations. Discrete Contin. Dyn. Syst. 10, 31-52 (2004)

3. Chepyzhov, W, Vishik, Ml: Attractors for Equations of Mathematical Physics. American Mathematical Society Colloquium Publications, vol. 49. Am. Math. Soc., Providence (2002)

4. Ghidaglia, JM, Temam, R: Attractors for damping nonlinear hyperbolic equations. J. Math. Pures Appl. 66, 273-319 (1987)

5. Haraux, A: Two remarks on dissipative hyperbolic problems. In: Lions, J-L (ed.) Séminaires de Collège de France (1984)

6. Haraux, A: Systèmes Dynamiques Dissipatifs et Applications. RMA, vol. 17. Masson, Paris (1991)

7. Hale, JK: Asymptotic Behavior and Dynamics in Infinite Dimensions. Pitman, London (1984)

8. Sell, GR, You, Y: Dynamics of Evolution Equations. Springer, New York (2002)

9. Temam, R: Infinite Dimensional Dynamical Systems in Mechanics and Physics. Springer, Berlin (1997)

10. Hale, JK, Raugel, G: Attractors for dissipative evolutionary equations. In: Perelló, C, Simó, C, Solà-Morales, J (eds.) Proceedings of the Conference EQUADIFF 91, Universitat de Barcelona, Barcelona, 26-31 August 1991, vol. 1, pp. 3-22 World Scientific, Singapore (1993)

11. Gallouët, T: Sur les injections entre espaces de Sobolev et espaces d'Orlicz et application au comportement à l'infini pour des équations des ondes semi-linéaires. Port. Math. 42,97-112 (1983/84)

12. Arrieta, JM, Carvalho, AN, Hale, JK: A damped hyperbolic equation with critical exponent. Commun. Partial Differ. Equ. $17,841-866$ (1992)

13. Ladyzhenskaya, O: Attractors for Semigroups and Evolution Equations. Cambridge University Press, Cambridge (1991)

14. Raugel, G: Global attractors in partial differential equations. In: Handbook of Dynamical Systems, vol. 2, pp. 885-982. North-Holland, Amsterdam (2002)

15. Robinson, C: Infinite-Dimensional Dynamical Systems: An Introduction to Dissipative Parabolic PDEs and the Theory of Global Attractors. Cambridge University Press, Cambridge (2001)

16. Ma, Q, Wang, S, Zhong, C: Necessary and sufficient conditions for the existence of global attractors for semigroups and applications. Indiana Univ. Math. J. 5(6), 1542-1558 (2002)

17. Pan, ZG, Pu, ZL, Ma, T: Global solutions to a class of nonlinear damped wave operator equations. Bound. Value Probl. 2012, $42(2012)$

18. Li, XS, Huang, NJ, O’Regan, D: Differential mixed variational inequalities in finite dimensional spaces. Nonlinear Anal. 72, 3875-3886 (2010)

19. Ma, T: Theories and Methods in Partial Differential Equations. Science Press, Beijing (2011)

20. Zhang, YH, Zhong, CK: Existence of global attractors for a nonlinear wave equation. Appl. Math. Lett. 18, 77-84 (2005) 